\title{
EFECTO DEL DESMANE INTENSIVO SOBRE EL DESARROLLO DEL RACIMO DE BANANO ${ }^{1}$
}

\author{
Alfonso Vargas-Calvo ${ }^{2}$
}

\begin{abstract}
RESUMEN
Efecto del desmane intensivo sobre el desarrollo del racimo de banano. El objetivo de este trabajo fue determinar la respuesta del racimo de banano a un desmane intensivo. El estudio se efectuó en el Caribe de Costa Rica del 2010 al 2011 en racimos de seis y ocho manos provenientes de racimos de nueve, diez y once (experimento 1) y de once, doce y trece manos (experimento 2). Mensualmente se midió en la segunda y quinta mano, el grosor y la distancia entre el ápice del fruto y el raquis y, a la cosecha, el peso del racimo, el grosor y el largo externo e interno del fruto, la distancia del ápice del fruto al raquis y se calculó, mediante la relación de ambos largos, el índice de curvatura. El grosor presentó un comportamiento lineal creciente y fue similar ( $\mathrm{P}>0,0508)$ entre desmanes en la mayoría de las evaluaciones de cada tamaño de racimo. La distancia tuvo una fase exponencial decreciente hasta la evaluación cuatro o cinco $y$, posteriormente se incrementó linealmente. No se observaron diferencias significativas $(\mathrm{P}>0,3797)$ para esta variable entre evaluaciones en los racimos de menor tamaño (nueve y diez manos, experimento 1) pero aumentó $(\mathrm{P}<0,0497)$ en aquellos con mayor desmane, aspecto que no alcanzó a ser significativo $(\mathrm{P}>0,0540)$ en algunas comparaciones. El intervalo floración-cosecha y el peso del racimo disminuyeron en los racimos más desmanados. A la cosecha, el grosor y el largo del fruto fueron similares $(\mathrm{P}>0,0732)$ entre desmanes y tamaños de racimo. La distancia solo varió en el experimento 2 donde fue mayor $(\mathrm{P}<0,0295)$ en los racimos más desmanados. El índice de curvatura no presentó diferencias $(\mathrm{P}>0,0645)$ en la mayoría de los casos.
\end{abstract}

Palabras clave: conformación de la mano, dimensiones del fruto, Musa AAA, peso del racimo, remoción de manos.

\begin{abstract}
Effect of intensive de-handing intensities on banana bunch development. The aim of this work was to study the response of banana bunches and fruits to extreme dehanding intensities.The study was performed from 2010 to 2011 in the Caribbean of Costa Rica in bunches with 6 and 8 fruit hands at harvest originating from bunches of 9 , 10 and 11 and 11, 12 and 13 hands for experiment 1 and 2, respectively. Thickness and distance of fruit tip to rachis of the second and fifth hand were measured at monthly intervals. At harvest, bunch weight, finger girth, external and internal fruit length, distance between fruit tip to rachis were measured, and the ratio between the two lengths was used to calculate the curvature index. Thickness linearly increased as fruit developed, being similar $(\mathrm{P}>0.0508)$ between hand trimming intensities in most evaluations. Distance from fruit tip to rachis had an exponential decreased until evaluations 4 or 5 and then linearly increased. There were no differences ( $\mathrm{P}>0.3797)$ for this variable on smaller bunches (9 and 10 hands, experiment 1) but increased $(\mathrm{P}<0.0497)$ in those with the highest trimming intensities. Some comparisons of this last variable among larger bunches were not significant $(\mathrm{P}>0.0540)$. The blooming-to-harvest interval and bunch weight decreased $(\mathrm{P}<0.0494)$ in bunches with higher hand trimming intensity. At harvest, thickness and fruit length were similar $(\mathrm{P}>0.0732)$ in all treatments regardless of bunch size. The distance varied only in experiment 2 which was higher $(\mathrm{P}<0.0295)$ in bunches with higher hand trimming intensities. The curvature ratio did not differ $(\mathrm{P}>0.0645)$ in most cases.
\end{abstract}

Keywords: hand conformation, fruit dimensions, Musa AAA, bunch weight, hand removal.

\footnotetext{
1 Recibido: 26 de setiembre, 2013. Aceptado: 18 de marzo, 2014. Proyecto de investigación "Manejo precosecha del racimo de banano". Corporación Bananera Nacional (CORBANA).

2 Corporación Bananera Nacional (CORBANA, S.A.). Limón, Costa Rica. Apdo. postal 506-2763-3055. alfvarga@corbana.co.cr
} 


\section{INTRODUCCIÓN}

La cosecha del racimo de banano para exportación se realiza considerando la edad fisiológica del mismo, así como el grosor del fruto central de la fila externa de la segunda mano basal, ambos preestablecidos y determinados fundamentalmente en función de la época del año y de las especificaciones (dimensiones) de los frutos de acuerdo con el mercado de destino (Jaramillo, 1982; Soto, 1992; Robinson y Galán-Sauco, 2010). Para cumplir dichas especificaciones, la eliminación de manos verdaderas es una práctica común en el racimo de banano (Jaramillo, 1982; Soto et al., 1992). Dicha labor de acuerdo con Soto et al. (1992) se realiza para cumplir con las exigencias de grosor y largo del fruto empacado y según Jaramillo (1982) con el propósito de aumentar la proporción de frutos con mejor presentación o calidad. No obstante, este último autor indica que la práctica tiene detractores y defensores.

Boncato (1969), Meyer (1975), Calvo (1984), Daniels et al. (1987), Irizarry et al. (1989), Irizarry et al. (1992), Irizarry et al. (1994), Johns (1996), Vargas et al. (1999), Mora (2000) y Vargas (2001) coinciden en que existe una disminución del peso del racimo conforme aumenta la intensidad del desmane. Esta reducción en peso no es compensada por un incremento en el peso de las manos remanentes en concordancia con lo descrito por Hasselbach e Idoe (1973), Meyer (1975), Vargas et al. (1999), Mora (2000), Vargas (2001) y Vargas (2002). Aumentos en la intensidad de desmane no han provocado el incremento de las dimensiones en los frutos remanentes, cuando la cosecha se realiza por edad y grosor del fruto central de la fila externa de la segunda mano basal (Vargas et al., 1999; Vargas y Blanco, 2000; Vargas 2001 y 2002). Estos resultados relacionados con el grosor y el largo del fruto, difieren de lo señalado por otros autores (Boncato, 1965; Calvo, 1984; Irizarry et al., 1992; Irizarry et al., 1994; Mora, 2000), quienes por el contrario, indican un efecto positivo de la labor. Sin embargo, dicha controversia podría ser explicada con base en la metodología de evaluación usada, donde en el primer caso (ausencia de efecto), las comparaciones (Vargas et al. 1999; Vargas y Blanco, 2000; Vargas, 2001 y 2002) se efectuaron entre grupos de racimos con igual número de manos a la floración y entre manos de igual posición en el racimo (racimos y manos equivalentes), mientras que en el segundo (presencia de efecto) las comparaciones se efectuaron entre grupos de racimos de diferente número de manos y de diferente posición en el racimo (racimos no equivalentes). Esta última metodología de acuerdo con Vargas y Blanco (2000, no debería usarse en trabajos de investigación relacionados con la eliminación de manos en el racimo de banano. Asimismo, otra particularidad que podría ayudar a explicar la diferencia entre resultados estaría básicamente dada por el sistema de cosecha. Estudios con diferentes intensidades de desmane en bananos del subgrupo Cavendish (Vargas 2001, 2002, 2008) indican que cuando se permite que los racimos de una misma edad lleguen en cosechas sucesivas (semanales) al calibre óptimo preestablecido de cosecha (sistema de cosecha para bananos de exportación), el beneficio real del desmane, al igual que lo indicado por otros autores (Daniells et al., 1987; Lara, 1970), no está en función de un aumento de las dimensiones del fruto sino por la reducción del período de desmane a cosecha. Este lapso de acuerdo con Vargas (2001) varía entre 0,7 a 1,8 días por mano eliminada y varía con la época climática y el cultivar.

Por el contrario, tal y como se sugiere en otros documentos (Vargas, 2003) cuando la cosecha se efectúa en racimos de una misma edad y en el mismo momento (cosecha por edad) el efecto de la remoción de frutos se expresaría básicamente en estos como un aumento de sus dimensiones. Este comportamiento diferencial asociado al sistema de cosecha fue corroborado por Vargas (2010) en plátanos del tipo Francés (FHIA-21) cuyo racimo presenta una distribución de manos y frutos similar a la de los bananos del subgrupo Cavendish.

Aumentos en la intensidad del desmane tampoco han resultado en una mayor separación de sus manos con respecto al raquis (Vargas, 2002), ni en el índice de curvatura del fruto (Vargas, 2002, 2003 y 2006). Sin embargo, Vargas (2006) reportó un aumento en la distancia del ápice del fruto central de la fila interna al raquis con las mayores intensidades de desmane.

Independientemente de la metodología de evaluación usada, todos los resultados y consideraciones antes mencionados fueron obtenidos mediante la aplicación de intensidades convencionales de desmane de uso comercial. No obstante, la respuesta del racimo a la labor bajo una práctica más rigurosa, ha sido poco evaluada.

El objetivo de este trabajo fue determinar la respuesta del racimo de banano a un desmane intensivo. 


\section{MATERIALES Y MÉTODOS}

\section{Localización, cultivar y densidades de población}

El estudio se realizó de febrero del 2010 a abril del 2011 en el Centro de Investigación Agrícola 28 Millas ( $10^{\circ} 5^{\prime} 52^{\prime}$ ' latitud Norte y $83^{\circ} 22^{\prime} 33$ " longitud Oeste) ubicado en el Caribe de Costa Rica en plantas de tercera generación del cv. Williams (experimento 1) y su sucesión o cuarta generación (experimento 2) que fueron sembradas en el año 2007 a una densidad de población de 2000 plantas/ha.

\section{Características de clima, suelo y nutrición mineral}

Registros climáticos del lugar (estación climática 28 Millas, CORBANA, S.A.) indican que en el año 2010 y 2011 la lluvia acumulada fue respectivamente de 3082 y $3025 \mathrm{~mm}$, con promedios de temperatura máxima de 31,7 y $31,4^{\circ} \mathrm{C}$; la temperatura mínima de 20,8 y $20,5^{\circ} \mathrm{C}$ y la humedad relativa de 93,2 y $91,6 \%$. El suelo es de origen sedimentario, textura franco arenoso (59,0\% arena; $6,0 \%$ arcilla y $35,0 \%$ limo) con las siguientes características: $\mathrm{pH}$ 6,29; materia orgánica $2,60 \%$ y un contenido de bases de: $\mathrm{Ca} 17,6 ; \mathrm{K} 0,33 ; \mathrm{Mg}$ $10,2 \mathrm{cmol} / 1$. La fertilización anual se realizó mediante la adición de $300 \mathrm{~kg}$ de N, $30 \mathrm{~kg}$ de $\mathrm{P}_{2} \mathrm{O}_{5}$ y $595 \mathrm{~kg}$ de $\mathrm{K}_{2} \mathrm{O}$, fraccionada en doce ciclos de cantidades iguales.

\section{Combate de Sigatoka negra y nematodos}

El combate de la Sigatoka negra (Mycosphaerella fijiensis) se realizó en todos los casos mediante la aspersión de mancozeb (1050 a $1200 \mathrm{~kg}$ i.a./ha) y difenbuconazole (100 g i.a./ha), bitertanol (150 g i.a./ ha), tridemorph (430 g i.a./ha) y axoxistrobina (100 g i.a./ha). Los mismos fueron aplicados en forma alternada y en emulsión con aceite mineral (5-10 l/ha) de acuerdo con los requerimientos y estrategias de combate propias de la época climática con una motobomba nebulizadora terrestre (Stihl@, modelo SR-400). Se utilizó como complemento al combate químico deshojas sanitarias semanales. El combate de nematodos se efectuó mediante la rotación cada cuatro meses de los nematicidas granulados fenamiphos, terbufos y ethoprofos, cada uno de ellos en dosis de $3 \mathrm{~g}$ de i.a. y en aplicación dirigida en un semicírculo desde la base de la planta hasta $30 \mathrm{~cm}$ de ella.

\section{Manejo precosecha del racimo}

El embolse se realizó una semana después de la floración (tipo prematuro) mediante fundas de polietileno de color azul de baja densidad $(12,7 \mu)$ y $81,3 \mathrm{~cm}$ de ancho, con perforaciones de $0,4 \mathrm{~mm}$ e impregnadas con el insecticida bifentrina $(0,1 \%)$. No se realizó desflora de los frutos. El desmane se efectuó una semana después del embolse y consideró la eliminación completa de manos verdaderas en conjunto con la falsa. Como mano verdadera se consideró aquel grupo de flores cuyos gineceos se desarrollaron normalmente. Como mano falsa se determinó al conjunto de flores en donde una o más de ellas se desarrollaron anormalmente, permaneciendo como una reminiscencia del ovario.

\section{Tratamientos y manejo experimental}

Se aplicaron dos intensidades de desmane a cada tamaño de racimo definido por la cantidad de manos totales verdaderas a la floración (MT) con el propósito de conformar en todos los casos, racimos de seis y ocho manos verdaderas presentes (MP). En el experimento 1 los tratamientos consistieron en la remoción de tres y una, de cuatro y dos, y de cinco y tres manos en racimos de nueve, diez y once manos verdaderas, respectivamente. En el experimento 2 la remoción de manos fue de cinco y tres, de seis y cuatro, y de siete y cinco manos en racimos de once, doce y trece manos (Cuadro 1).

Los tratamientos se asignaron de acuerdo con el tamaño (MT) de cada racimo y en función de su ocurrencia en la plantación. Cada planta y su racimo se consideraron como una repetición. El número de repeticiones para cada MT de acuerdo con la cantidad de manos presentes definidas ( $\mathrm{MP}=6$ y 8 ) en todos los casos por el desmane fue de 22 y 16 para MT 9, de 23 y 28 para MT 10 y de 30 y 31 para MT 11 (experimento 1$)$ y de 24 y 22 para MT 11 , de 28 y 22 para MT 12 y de 16 y 16 para MT 13 (experimento 2).

\section{Variables medidas}

Las variables medidas semanalmente (manos segunda y quinta en todos los tamaños de racimo e intensidad de desmane) a partir de la segunda semana de emitida la inflorescencia y por doce semanas más, así como a la cosecha fueron: grosor del fruto central 
Cuadro 1. Desglose de tratamientos de acuerdo con la cantidad de manos totales verdaderas a la floración (MT) y la intensidad de desmane para la retención en todos los casos de seis y ocho manos verdaderas presentes (MP). Matina, Limón, Costa Rica. 2010-2011.*

\begin{tabular}{cccc}
\hline $\begin{array}{c}\text { Manos verdaderas totales } \\
\text { (MT) a la floración }\end{array}$ & $\begin{array}{c}\text { Manos verdaderas eliminadas } \\
\text { (ME) por el desmane }\end{array}$ & $\begin{array}{c}\text { Manos verdaderas presentes } \\
\text { (MP) luego del desmane }\end{array}$ \\
\hline Experimento 1 & 9 & 3 & 6 \\
& 9 & 1 & 8 \\
& & 4 & 6 \\
& 10 & 2 & 8 \\
& 10 & & 6 \\
& 11 & 5 & 8 \\
11 & 3 & 6 \\
Experimento 2 & & 8 \\
& 11 & 5 & 6 \\
11 & 3 & 8 \\
12 & & 6 \\
12 & 6 & 8 \\
\hline 13 & 4 & 6 \\
\hline
\end{tabular}

* El número de observaciones fue para MP 6 y 8 de 22 y 16 para MT 9, de 23 y 28 para MT 10 y de 30 y 31 para MT 11 (experimento 1) y de 24 y 22 para MT 11, de 28 y 22 para MT 12 y de 16 para MT 13 (experimento 2).

de la fila externa (medido en $\mathrm{mm}$ y presentado en treintaidosavos de pulgada, donde un treintaidosavo de pulgada equivale a $0,794 \mathrm{~mm}$ ) y distancia $(\mathrm{cm})$ del fruto central de la fila interna al raquis medida en un ángulo de $90^{\circ}$ con la ayuda de una regla graduada en $\mathrm{mm}$. Además para cada tamaño de racimo e intensidad, se midieron a la cosecha, el peso $(\mathrm{kg})$ del racimo y el largo (cm de pulpa a punta) externo e interno del fruto central de la fila externa así como la distancia $(\mathrm{cm})$ del fruto central de la fila interna al raquis de la segunda y quinta mano. A partir de la relación entre el largo interno y externo, se determinó el índice de curvatura $(\mathrm{Ic}=\mathrm{Li} / \mathrm{Le})$ del fruto en las manos antes citadas, donde el valor de 1 significa un fruto recto.

\section{Desarrollo y cosecha de los racimos}

El desarrollo de los racimos transcurrió desde febrero a octubre del 2010 en el experimento 1 y de agosto del 2010 a abril del 2011 en el experimento 2 .
En consecuencia, los racimos de ambos experimentos se desarrollaron parte en época climática adversa y parte en época climática favorable. De acuerdo con el conocimiento climático de la región, esto comprende cuatro épocas (Vargas y Blanco, 2004): 1- noviembre diciembre y enero (fría y lluviosa), 2- febrero, marzo y abril (moderadamente caliente y seca), 3- mayo, junio y julio (caliente y lluviosa) y agosto, setiembre y octubre (caliente y seca). Las épocas 1 y 2 se consideran como adversas mientras que las 3 y 4 como favorables para el crecimiento de la planta y el racimo del banano. La cosecha se efectuó cuando estos presentaron en el fruto central de la fila externa de la segunda mano, un grosor preestablecido de $39,34 \mathrm{~mm}$ (44,0 treintaidosavos de pulgada).

\section{Análisis de los datos}

Con los datos de las evaluaciones semanales, se construyó una curva de crecimiento para las variables 
del fruto en ambos tamaños de racimo (seis y ocho manos remanentes) que se conformaron a partir de las diferentes intensidades de desmane. En cada evaluación se comparó la media obtenida de las variables del fruto para cada tamaño de racimo (MT) y desmane o manos presentes (MP). Dichas comparaciones se hicieron para cada experimento mediante la aplicación de un modelo factorial de tres niveles de MT (nueve, diez y once manos en el experimento $1 \mathrm{y}$ once, doce y trece para el experimento 2) por dos niveles de MP (seis y ocho para ambos experimentos). El análisis se efectuó con el Proc Mixed de SAS, aplicando un modelo de covarianza autoregresivo de primer orden a las mediciones repetidas de los frutos. Los datos a cosecha se analizaron para cada experimento mediante un modelo factorial de tres niveles de MT x dos niveles de MP y comparaciones de niveles de cada sector. Se utilizó el Proc GLM de SAS (SAS Institute, 2004).

\section{RESULTADOS Y DISCUSIÓN}

\section{Medición manual del grosor del fruto central de la fila externa}

El grosor del fruto central de las manos seleccionadas (segunda y quinta), independientemente de la intensidad de desmane, presentó un comportamiento lineal creciente durante el desarrollo del racimo en todos los tamaños de ambos experimentos (Figuras 1 y 2).

En el experimento 1 (Figura 1) no hubo diferencias $(\mathrm{P}>0,0529)$ en el grosor entre intensidades de desmane en la mayoría de las evaluaciones para los tamaños de racimo considerados. No obstante, en aquellos donde estas se expresaron, el mayor valor se observó con el desmane más intenso. Esto sucedió en la segunda mano $(\mathrm{P}<0,0475)$ para el racimo de nueve manos (onceava evaluación) y para el racimo de once manos (novena a onceava evaluación) y en la quinta mano $(\mathrm{P}<0,0468)$ para el racimo de nueve manos (novena evaluación), de diez manos (doceava evaluación) y de once manos (sétima a décima y doceava evaluación). En el experimento 2 (Figura 2), no hubo diferencias $(\mathrm{P}>0,0551)$ en el grosor entre intensidades de desmane en la mayoría de las evaluaciones para los tamaños de racimo considerados. Sin embargo, cuando estas ocurrieron, el mayor valor se determinó con la mayor remoción de manos. Esto sucedió en la segunda mano $(\mathrm{P}<0,0476)$ para el racimo de once manos (sétima a novena y onceava evaluación) y de doce manos (cuarta a sexta evaluación) y en la quinta mano $(\mathrm{P}<0,0469)$ para el racimo de once manos (octava, novena y onceava evaluación), de doce manos (tercera a sexta, novena y onceava evaluación) y de trece manos (novena evaluación).

Al respecto, Vargas (2001) encontró que el índice de crecimiento diario del fruto en grosor aumentó linealmente con la intensidad del desmane, condición que se observó en este estudio, particularmente en las etapas tardías del desarrollo del racimo. Sin embargo, cuando la cosecha se realiza con el criterio de un grosor mínimo de fruto se da oportunidad de que los racimos menos desmanados alcancen, aunque en un mayor tiempo, el mismo grosor de fruto preestablecido que aquellos más desmanandos, en concordancia con lo indicado anteriormente por dicho autor. A pesar del uso de intensidades extremas de desmane, la expresión de las diferencias entre desmanes estarían dadas a la cosecha básicamente por una reducción del intervalo floración-cosecha y no por un aumento en las dimensiones del fruto, tal y como se ha sugerido en otros estudios (Vargas, 2001, 2002 y 2006).

\section{Medición mensual de la distancia del fruto central de la fila interna}

La distancia del ápice del fruto central al raquis de las manos evaluadas tuvo una fase exponencial decreciente hasta la evaluación 4 o 5 y de ahí aumentó linealmente (Figuras 3 y 4). Esta variable fue similar entre intensidades de desmane $(\mathrm{P}>0,3797)$ para todas las evaluaciones de la segunda mano en los racimos de nueve y diez manos del experimento 1 (Figura 3 ). También fue similar en las primeras tres evaluaciones del racimo con once manos ( $\mathrm{P}>0,0897)$, a partir de la cual aumentó $(\mathrm{P}<0,0103)$ con el mayor desmane. En la quinta mano las diferencias entre intensidades de desmane $(\mathrm{P}<0,0490)$ se expresaron solo de la cuarta a la sétima y novena evaluación en los racimos de once manos donde la distancia aumentó en aquellos más desmanados. En el experimento 2 (Figura 4) hubo diferencias para la segunda mano entre desmanes $(\mathrm{P}<$ 0,0328 ) en las evaluaciones primera, tercera a quinta y sétima a doceava en el racimo de once manos. En la quinta mano las diferencias entre desmane se produjeron solo en las evaluaciones octava del racimo 

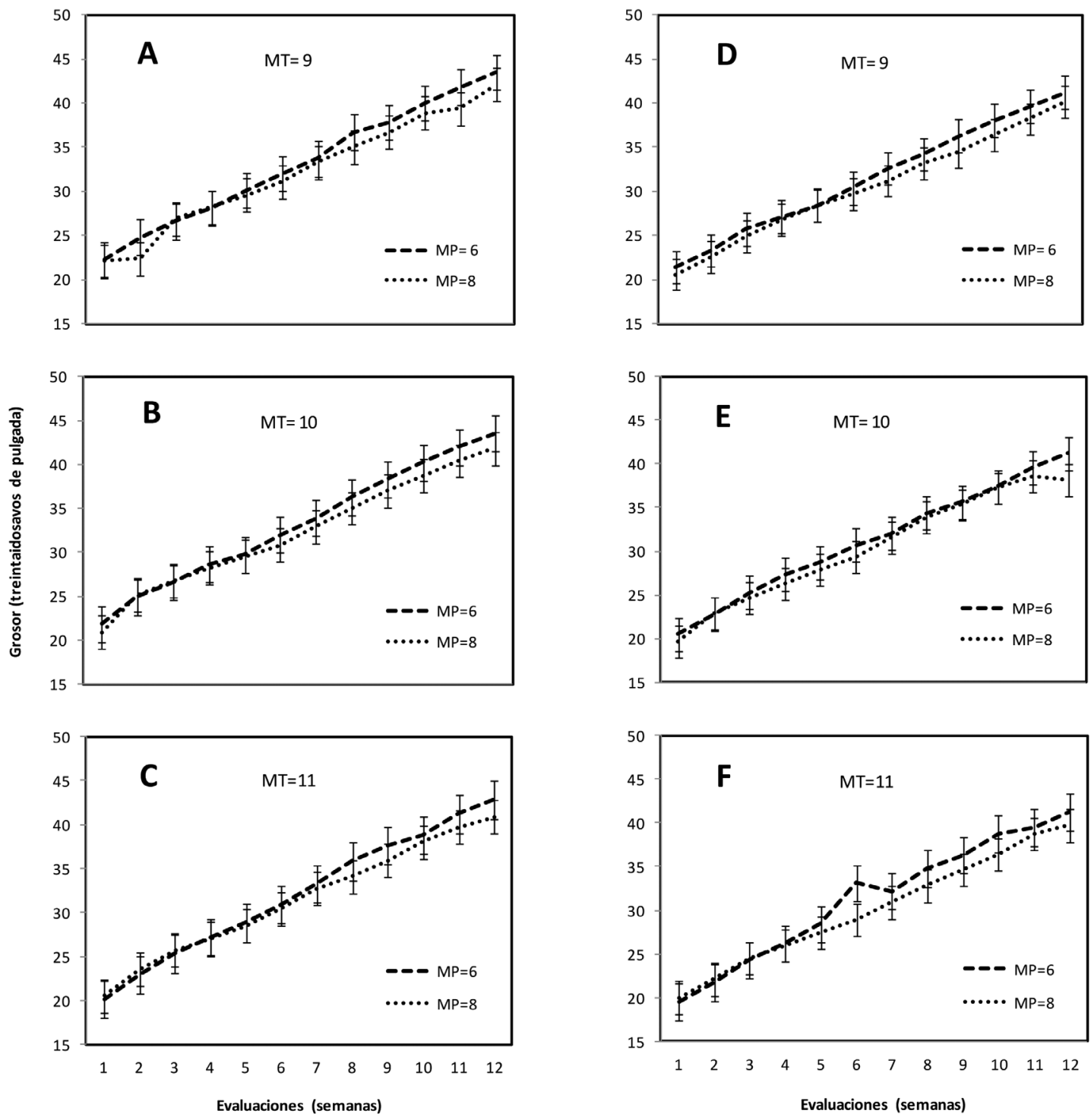

Figura 1. Variación en el tiempo del grosor (treintaidosavos de pulgada) del fruto central de la fila externa de la segunda (A, B y C) y quinta (D, E y F) mano en racimos de seis y ocho manos presentes (MP) provenientes del desmane de racimos de nueve, diez y once manos totales (MT) a la floración. Experimento 1. Matina, Limón, Costa Rica. 2010-2011.

de once manos $(\mathrm{P}=0,0439)$, y quinta y novena del racimo de doce manos $(\mathrm{P}<0,0497)$, con un aumento de la distancia, en todos los casos, con el mayor desmane.

A pesar de que en muchos casos, las diferencias entre intensidades de desmane para esta variable entre evaluaciones no alcanzaron a ser significativas, la magnitud de las mismas indica una mayor distancia o separación entre la mano y el raquis en los racimos de mayor tamaño con la intensidad más alta de desmane. Lo anterior sugiere que existe una respuesta diferencial según tamaño del racimo y la intensidad de desmane. Racimos con menos manos verdaderas a la floración y rangos menores de desmane, presentarían una nula o escasa respuesta a este y viceversa. 

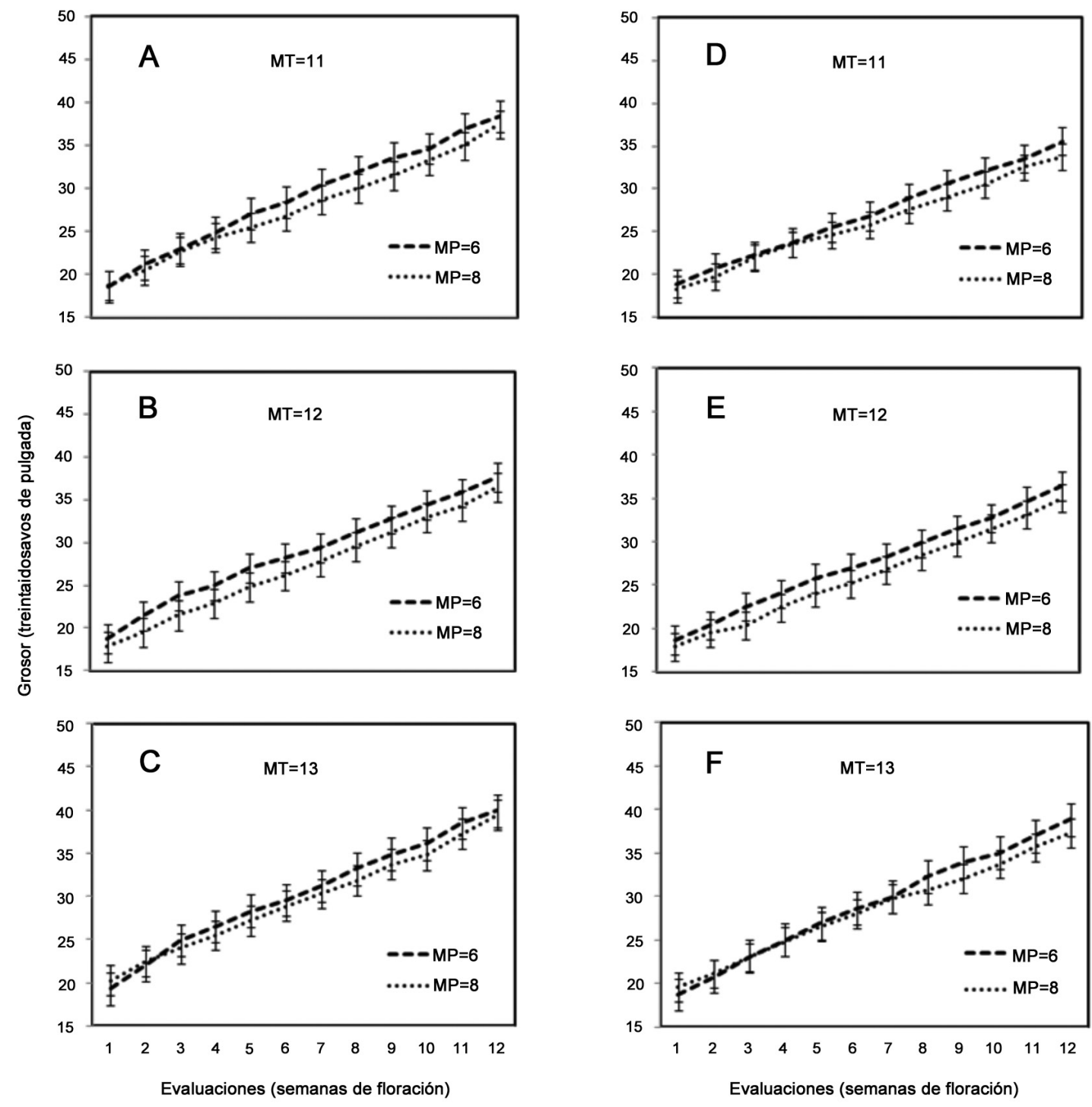

Figura 2. Variación en el tiempo del grosor (treintaidosavos de pulgada) del fruto central de la fila externa de la segunda (A, B y C) y quinta (D, E y F) mano en racimos de seis y ocho manos presentes (MP) provenientes del desmane de racimos de once, doce y trece manos totales (MT) a la floración. Experimento 2. Matina, Limón, Costa Rica. 2010-2011.

\section{Medición a la cosecha del desarrollo y peso del racimo}

El número de días del desmane a la cosecha (Cuadro 2) se redujo en los racimos con mayor remoción de manos, aspecto que, sin embargo, solo alcanzó a ser significativo en aquellos de once manos del experimento $1(\mathrm{P}=0,0010)$ y en los de doce manos del experimento $2(\mathrm{P}=0,0164)$. No obstante, cuando el conjunto de datos de cada experimento se analizó ignorando el tamaño de racimo (interacción MT x MP: $\mathrm{P}=0,2117$ y $\mathrm{P}=0,3741$; respectivamente) la reducción en el intervalo floración - cosecha producto de la mayor intensidad de desmane fue confirmada y 

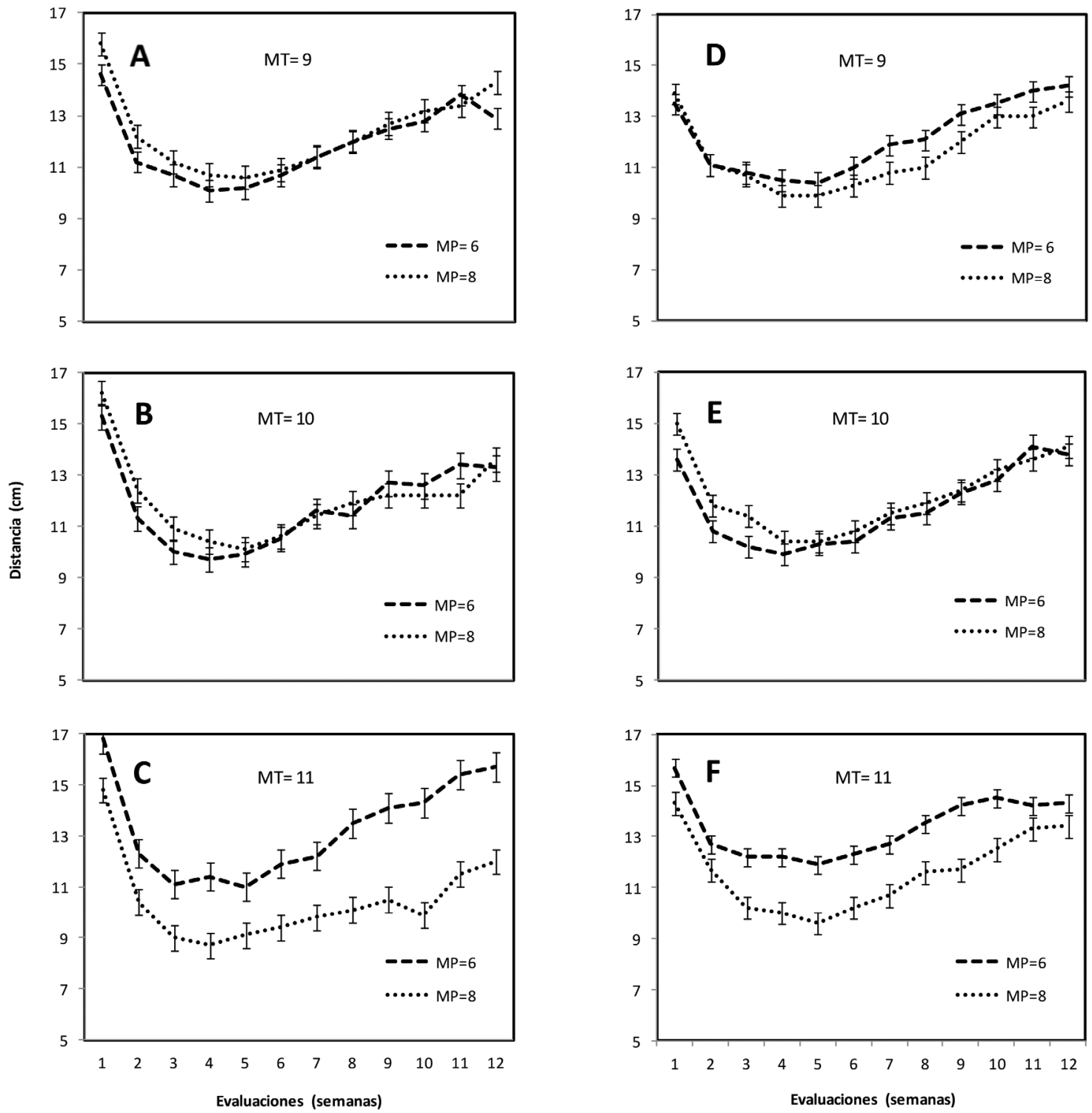

Figura 3. Variación en el tiempo de la distancia $(\mathrm{cm})$ entre el ápice del fruto central de la fila interna y el raquis de la segunda (A, B y C) y quinta (D, E y F) mano en racimos de seis y ocho manos presentes (MP) provenientes del desmane de racimos de nueve, diez y once manos totales (MT) a la floración. Experimento 1. Matina, Limón, Costa Rica. 2010-2011.

estadísticamente respaldado tanto para el experimento $1(\mathrm{P}=0,0042)$ como en el experimento $2(\mathrm{P}=0,0494)$.

El peso del racimo (Cuadro 2) disminuyó en los racimos con mayor remoción de manos y fue significativo en el experimento 1 para el racimo de nueve manos $(\mathrm{P}=0,0438)$. En el experimento 2, el peso disminuyó $(\mathrm{P}<0,0313)$ con la mayor intensidad del desmane en todos los tamaños de racimo. Cuando se ignoró el tamaño de este (interacción MT x MP; P= 0,8562 y $\mathrm{P}=0,5475$, respectivamente) la reducción en 

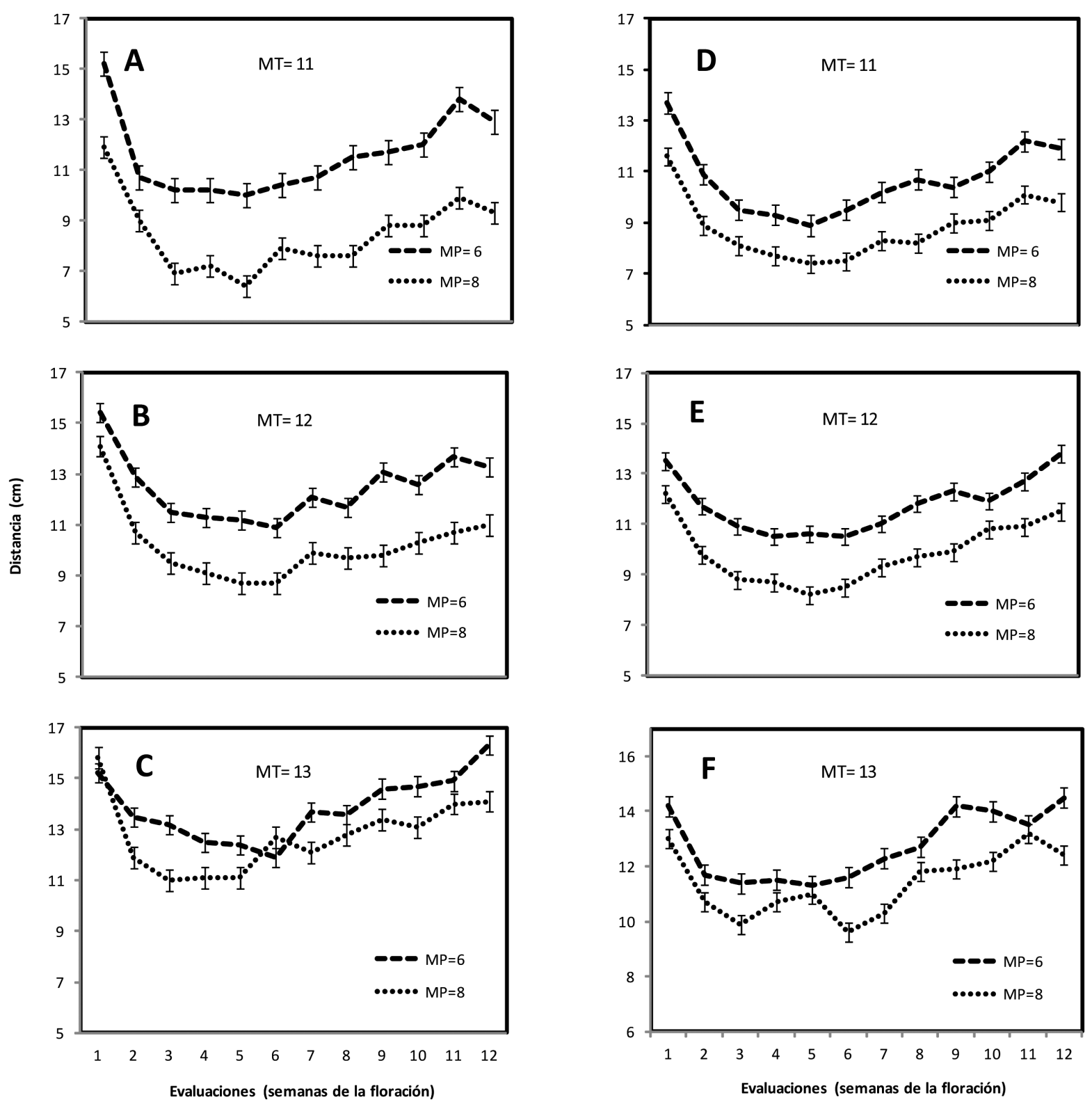

Figura 4. Variación en el tiempo de la distancia $(\mathrm{cm})$ entre el ápice del fruto central de la fila interna y el raquis de la segunda (A, B y C) y quinta (D, E y F) mano en racimos de seis y ocho manos presentes (MP) provenientes del desmane de racimos de once, doce y trece manos totales (MT) a la floración. Experimento 2. Matina, Limón, Costa Rica. 2010-2011.

peso, producto de la mayor intensidad de desmane, fue nuevamente confirmada para el experimento $1(\mathrm{P}=$ $0,0015)$ y el experimento $2(\mathrm{P}=0,0001)$.

La disminución en el período de desarrollo del racimo y la reducción de su peso con la mayor intensidad de desmane coincide con los resultados de otros autores (Hasselbach e Idoe, 1973; Meyer, 1975; Vargas, 2001 y 2002), aspecto que también ha sido determinado en plátanos del tipo Francés (Irizarry et al., 1991; Rodríguez et al., 2006; Vargas, 2010) con una 
Cuadro 2. Número de días de la floración a la cosecha, peso del racimo y dimensiones del fruto de manos seleccionadas en racimos de banano (Musa AAA) de varios tamaños y desmanados a seis y ocho manos presentes. Matina, Limón, Costa Rica. 2010-2011.

\begin{tabular}{|c|c|c|c|c|c|c|c|c|}
\hline \multirow{2}{*}{$\begin{array}{l}\text { Manos } \\
\text { totales }\end{array}$} & \multirow{2}{*}{$\begin{array}{c}\text { Manos } \\
\text { eliminadas } \\
\end{array}$} & \multirow{2}{*}{$\begin{array}{c}\text { Manos } \\
\text { presentes }\end{array}$} & \multirow{2}{*}{$\begin{array}{l}\text { Días a la } \\
\text { cosecha }^{1}\end{array}$} & \multirow{2}{*}{$\begin{array}{c}\text { Peso del } \\
\text { racimo }(\mathrm{kg})\end{array}$} & \multicolumn{2}{|c|}{ Segunda mano } & \multicolumn{2}{|c|}{ Quinta mano } \\
\hline & & & & & Grosor $^{2}$ & Largo $^{3}$ & Grosor & Largo \\
\hline \multicolumn{9}{|c|}{ Experimento 1} \\
\hline 9 & 3 & 6 & 93,9 & 18,8 & 44,5 & 24,0 & 41,7 & 21,2 \\
\hline 9 & 1 & 8 & 97,4 & 21,7 & 44,2 & 23,1 & 41,5 & 20,9 \\
\hline Probabilidad & & & 0,3039 & 0,0438 & 0,5144 & 0,2730 & 0,8065 & 0,6889 \\
\hline 10 & 4 & 6 & 94,5 & 23,6 & 44,9 & 25,1 & 42,4 & 22,2 \\
\hline 10 & 2 & 8 & 97,1 & 25,7 & 44,3 & 24,3 & 42,2 & 22,0 \\
\hline Probabilidad & & & 0,3500 & 0,0680 & 0,4764 & 0,2109 & 0,7032 & 0,7689 \\
\hline 11 & 5 & 6 & 91,6 & 24,5 & 44,3 & 24,7 & 42,4 & 22,7 \\
\hline 11 & 3 & 8 & 100,5 & 26,4 & 44,1 & 24,3 & 42,2 & 22,6 \\
\hline Probabilidad & & & 0,0010 & 0,0755 & 0,0732 & 0,4658 & 0,6954 & 0,8119 \\
\hline MT x MP $(\mathrm{Pr}$ & abilidad) & & 0,2117 & 0,8562 & 0,7371 & 0,8873 & 0,9987 & 0,9844 \\
\hline $\mathrm{MP}=6$ & & & 93,3 & 22,3 & 44,5 & 24,6 & 42,2 & 22,0 \\
\hline $\mathrm{MP}=8$ & & & 98,3 & 24,6 & 44,2 & 23,9 & 42,0 & 21,8 \\
\hline Probabilidad & & & 0,0042 & 0,0015 & 0,3345 & 0,0766 & 0,5694 & 0,5338 \\
\hline \multicolumn{9}{|c|}{ Experimento 2} \\
\hline 11 & 5 & 6 & 101,7 & 19,2 & 43,4 & 22,7 & 40,7 & 20,6 \\
\hline 11 & 3 & 8 & 103,6 & 22,1 & 43,3 & 22,3 & 40,7 & 20,5 \\
\hline Probabilidad & & & 0,4813 & 0,0008 & 0,5201 & 0,1679 & 0,9824 & 0,8330 \\
\hline 12 & 6 & 6 & 105,4 & 20,7 & 43,4 & 22,3 & 40,2 & 20,5 \\
\hline 12 & 4 & 8 & 112,1 & 23,0 & 43,3 & 21,9 & 40,8 & 20,1 \\
\hline Probabilidad & & & 0,0164 & 0,0313 & 0,3966 & 0,3205 & 0,1392 & 0,2308 \\
\hline 13 & 7 & 6 & 99,8 & 22,2 & 43,8 & 23,0 & 41,2 & 20,9 \\
\hline 13 & 5 & 8 & 101,4 & 25,9 & 43,5 & 22,2 & 40,7 & 20,5 \\
\hline Probabilidad & & & 0,6992 & 0,0051 & 0,2204 & 0,1644 & 0,2867 & 0,2903 \\
\hline MT x MP (Pr & abilidad) & & 0,3741 & 0,5475 & 0,8780 & 0,8544 & 0,2045 & 0,6888 \\
\hline $\mathrm{MP}=6$ & & & 102,3 & 20,7 & 43,5 & 22,7 & 40,7 & 20,7 \\
\hline $\mathrm{MP}=8$ & & & 105,7 & 23,9 & 43,4 & 22,0 & 40,7 & 20,3 \\
\hline Probabilidad & & & 0,0494 & 0,0001 & 0,1121 & 0,0284 & 0,9403 & 0,1606 \\
\hline
\end{tabular}

${ }^{1 /}$ Medidos desde el desmane. ${ }^{2 /}$ medido en mm y presentado en treintaidosavos de pulgada ( 1 treintaidosavo de pulgada $=0,794$ $\mathrm{mm}) .{ }^{3 /} \mathrm{cm}$ de pulpa a punta.

conformación de manos (doble hilera de frutos) similar al racimo de banano. La reducción en peso del racimo, independientemente de que estos sean cosechados por edad (preestablecida) o por edad y grosor (ambos preestablecidos) del fruto central de la segunda mano, indica que el peso perdido por las manos removidas no se restituye luego en el peso final de las manos remanentes. Por ello, la eliminación de manos debe 
efectuarse de manera racional, descartando solamente aquellas que no cumplan los requisitos mínimos de grosor y longitud de sus frutos o gajos.

\section{Medición a la cosecha de las dimensiones del fruto y de la distancia entre el ápice y el raquis. Estima- ción del índice de curvatura}

El grosor y el largo (Cuadro 2) del fruto central de la fila externa de la segunda y quinta mano basal no difirieron (grosor: $\mathrm{P}>0,0732$; largo: $\mathrm{P}>0,0766$ ) con las intensidades de desmane en ninguno de los experimentos ni con los tamaños de racimo. Sin embargo, cuando en el experimento 2 se ignoró el tamaño del racimo (interacción MT x MP=0,8544), aquellos con mayor desmane presentaron frutos más largos $(\mathrm{P}=0,0284)$. A pesar de tal diferencia, y dado que la cosecha de los racimos se efectuó con un grosor preestablecido ( 44,0 treintaidosavos de pulgada) en el fruto central de la segunda mano, las dimensiones del fruto y su respuesta al desmane se expresaron básicamente en un menor tiempo de desmane a cosecha, y no como un aumento de las dimensiones de los frutos remanentes. Esta reducción del intervalo como respuesta del racimo a la remoción de manos también ha sido señalada tanto en banano (Vargas, 2001, 2002 y 2003) como en plátanos del tipo Francés (Vargas, 2010).

La distancia (Cuadro 3) del ápice del fruto central de la fila interna al raquis, no difirió en el experimento 1 entre intensidades de desmane para la segunda y quinta mano evaluadas, tanto cuando se consideró $(\mathrm{P}>0,0732)$ como cuando se ignoró $(\mathrm{P}>0,3345)$ el tamaño (interacción MT x MP: P $>0$,2152) del racimo. En el experimento 2, la distancia aumentó en todos los tamaños de racimo con el mayor desmane pero solo alcanzó a ser significativa en la segunda mano para los racimos de once y doce manos $(\mathrm{P}<0,0352)$ $\mathrm{y}$ en la sexta mano para el racimo de once manos $(\mathrm{P}=$ $0,0336)$. Cuando en este experimento se ignoró el tamaño de racimo (interacción MT x MP; $\mathrm{P}=0,6140$ ) el aumento en la distancia se confirmó tanto para la segunda $(\mathrm{P}<0,0055)$ como para la quinta $(\mathrm{P}<0,0295)$ mano. El índice de curvatura (Cuadro 3), excepto por el menor valor de la segunda mano en los racimos de once manos con mayor desmane $(\mathrm{P}<0,0115)$ no presentó diferencias en ambos experimentos tanto cuando se consideró $(\mathrm{P}>0,0645)$ o se ignoró $(\mathrm{P}>0,1915)$ el tamaño del racimo.
A pesar de que en la mayoría de los tamaños de racimo y manos evaluadas a la cosecha, no hubo diferencias estadísticas en la distancia del fruto central al raquis, se observó en ambos experimentos, y particularmente con los racimos de mayor cantidad de manos (experimento 2), un aumento de la distancia con la mayor intensidad de desmane. No obstante, cuando se consideró la interacción y fue posible ignorar uno de los factores, tamaño de racimo (MT), fue posible verificar el aumento de la distancia con la mayor intensidad de desmane en la segunda y quinta mano. Este comportamiento es congruente al señalado por Vargas (2006) con el cv. Valery donde el autor encontró que las mayores intensidades de desmane constituidas por dos, tres y cuatro manos eliminadas $(9,10$ y 11 MT, respectivamente) aumentaron la distancia entre la mano y el raquis en comparación con las menores intensidades (una, dos y tres manos removidas, respectivamente).

En contraste, Vargas (2002) determinó que dicha variable no varió entre intensidades de desmane en racimos del cv. Grande Naine de diez y once manos verdaderas con dos y tres manos removidas, respectivamente, y en racimos de doce y trece manos verdaderas con tres y cuatro manos removidas, mientras que en el cv. Williams ello sucedió para la segunda y última mano comparable de racimos de nueve y diez manos verdaderas con dos y tres removidas, respectivamente, excepto para la última mano comparable en los racimos de nueve manos.

Esta asimetría entre resultados de distancia podría estar dada en función de la intensidad de desmane que se efectuó en uno u otro experimento para cada tamaño de racimo. En el presente estudio, dicha práctica consideró una remoción de manos mayor a la que generalmente se realiza bajo condiciones comerciales. Comparando racimos de once, doce y trece manos verdaderas, Vargas (2002) removió un 22 y 30\% para baja y alta intensidad de desmane, mientras en el presente estudio, con el mismo tamaño de racimos, la baja y alta intensidad de desmane removió un 34 y $51 \%$ de la biomasa total del racimo. Vargas (2002), obtuvo una diferencia de un $8 \%$ de biomasa entre intensidades de desmane, pero en el presente estudio fue de $17 \%$.

Esta diferencia en biomasa removida no repercutió en la expresión de las otras variables de producción, incluido el índice de curvatura del fruto y se atribuye al similar criterio de cosecha por grosor 
Cuadro 3. Distancia del fruto central de la fila interna al raquis e índice de curvatura del fruto central de la fila externa de manos seleccionadas en racimos de banano (Musa AAA) de varios tamaños y desmanados a seis y ocho manos presentes. Matina, Limón, Costa Rica 2010-2011.

\begin{tabular}{|c|c|c|c|c|c|c|}
\hline \multirow[t]{2}{*}{ Manos totales } & \multirow{2}{*}{$\begin{array}{c}\text { Manos } \\
\text { eliminadas }\end{array}$} & \multirow{2}{*}{$\begin{array}{c}\text { Manos } \\
\text { presentes }\end{array}$} & \multicolumn{2}{|c|}{ Segunda mano } & \multicolumn{2}{|c|}{ Quinta mano } \\
\hline & & & Distancia $^{1}$ & Curvatura $^{2}$ & Distancia & Curvatura \\
\hline \multicolumn{7}{|c|}{ Experimento 1} \\
\hline 9 & 3 & 6 & 14,7 & 1,55 & 14,9 & 1,53 \\
\hline 9 & 1 & 8 & 13,4 & 1,49 & 14,3 & 1,58 \\
\hline Probabilidad & & 0,5144 & 0,2383 & 0,6832 & 0,2044 & \\
\hline 10 & 4 & 6 & 12,2 & 1,54 & 13,8 & 1,57 \\
\hline 10 & 2 & 8 & 13,3 & 1,52 & 14,4 & 1,52 \\
\hline Probabilidad & & 0,4764 & 0,6226 & 0,5879 & 0,0932 & \\
\hline 11 & 5 & 6 & 15,3 & 1,46 & 15,8 & 1,51 \\
\hline 11 & 3 & 8 & 12,6 & 1,56 & 14,9 & 1,53 \\
\hline Probabilidad & & 0,0732 & 0,0115 & 0,4082 & 0,5681 & \\
\hline \multicolumn{3}{|c|}{ MT x MP (Probabilidad) } & 0,2152 & 0,0251 & 0,611 & 0,0952 \\
\hline \multicolumn{3}{|l|}{$\mathrm{MP}=6$} & 14,1 & 1,52 & 14,8 & 1,54 \\
\hline \multicolumn{3}{|l|}{$\mathrm{MP}=8$} & 13,1 & 1,52 & 14,5 & 1,54 \\
\hline \multicolumn{7}{|c|}{ Experimento 2} \\
\hline 11 & 5 & 6 & 13,3 & 1,51 & 13,6 & 1,55 \\
\hline 11 & 3 & 8 & 9,5 & 1,59 & 11,1 & 1,58 \\
\hline \multicolumn{2}{|l|}{ Probabilidad } & 0,0210 & 0,0645 & 0,0336 & 0,4849 & \\
\hline 12 & 6 & 6 & 15,1 & 1,55 & 13,9 & 1,52 \\
\hline 12 & 4 & 8 & 11,8 & 1,55 & 12,8 & 1,53 \\
\hline \multicolumn{2}{|l|}{ Probabilidad } & 0,0352 & 0,8217 & 0,3467 & 0,7769 & \\
\hline 13 & 7 & 6 & 16,2 & 1,51 & 14,9 & 1,56 \\
\hline 13 & 5 & 8 & 14,9 & 1,53 & 13,8 & 1,54 \\
\hline \multicolumn{2}{|l|}{ Probabilidad } & 0,4755 & 0,7753 & 0,4089 & 0,5961 & \\
\hline \multicolumn{3}{|c|}{ MT x MP (Probabilidad) } & 0,8780 & 0,4666 & 0,6140 & 0,6899 \\
\hline \multicolumn{3}{|l|}{$\mathrm{MP}=6$} & 14,9 & 1,52 & 14,1 & 1,54 \\
\hline \multicolumn{3}{|l|}{$\mathrm{MP}=8$} & 12,0 & 1,55 & 12,6 & 1,55 \\
\hline \multicolumn{3}{|l|}{ Probabilidad } & 0,0055 & 0,1915 & 0,0295 & 0,8595 \\
\hline
\end{tabular}

${ }^{1 /}$ Distancia en $\mathrm{cm}$ del ápice del fruto central de la fila interna al raquis medida en un ángulo de $90^{\circ} .{ }^{2 /}$ Relación entre el largo externo y el largo interno del fruto central de la fila externa, en donde IC=1 significa un fruto recto.

preestablecido en ambos estudios. Sin embargo, el más rápido crecimiento de los frutos con las mayores intensidades de desmane y fundamentalmente bajo la particular intensidad de remoción de manos que se utilizó, provocó una mayor apertura de la mano con respecto al raquis.

A pesar de que algunas de las intensidades de desmane, particularmente aquellas extremas, no constitui- 
rían en la actualidad una opción comercial de manejo, su estudio permitió ampliar el conocimiento que sobre la práctica expresan el racimo de banano (Musa AAA, cv. Williams) y sus frutos. Los resultados obtenidos permiten confirmar la necesidad de racionalizar la labor de desmane considerando fundamentalmente, además de los criterios establecidos de cosecha, el tamaño del racimo, la época climática, el destino del mercado y las dimensiones de calidad del fruto tal y como lo indican Vargas y Blanco (2004). Asimismo, también se debe reafirmar que el efecto real del desmane al igual que lo indicado por otros autores (Lara, 1970; Vargas, 2001; Vargas, 2002) está básicamente dado por una reducción del intervalo desmane-cosecha y no por un aumento de las dimensiones del fruto, reducción que sin embargo, especialmente, bajo condiciones adversas de clima o de alta severidad de la Sigatoka negra, sería una herramienta valiosa como parte de las estrategias de manejo de la plantación.

\section{LITERATURA CITADA}

Boncato, A. 1969. Effects of reducing the number of hands in a bunch of Lacatan banana. Journal of Plant Industry 32(3-4):243-251.

Calvo, J. 1984. Efecto del desmane en la calidad del fruto del banano (Musa AAA, Sub grupo Cavendish 'Gran enano'). Tesis Lic. Ing. Agr., Universidad de Costa Rica, San José, Costa Rica.

Daniells, J., P. O'Farrel, C. Mulder, y S. Campbell. 1987. Effects of bunch covering and bunch trimming on bananas in north Queensland. Queensland Journal Of Agricultural and Animal Sciences 44(2):101-105.

Hasselbach, O., y J. Idoe. 1973. Dehanding of bananas in Surinam. Surinaamse Landbouw 21:127-132.

Irizarry, H., E. Rivera, A. Krikorian, y J. Rodríguez. 1991. Proper bunch management of the French-type superplantain (Musa acuminata x M. Balbisiana) in Puerto Rico. The Journal of Agriculture of University of Puerto Rico 75(2):163-171.

Irizarry, H., E. Hernández, y J. Rodríguez. 1992. Bunch and ratoon management for profitable production of high quality bananas (Musa acuminata, AAA). The Journal of Agriculture of University of Puerto Rico 76(3-4):119-129.

Irizarry, H., E. Hernández, y J. Rodríguez. 1994. Yield of five dwarf banana cultivars grown with minimun tillage in Puerto Rico's mountain region. The Journal of Agriculture of University of Puerto Rico 78(1-2):17.

Irizarry, H., E. Hernández, E. Rivera, E. De Caloni, y R. Guadalupe. 1989. Perfomance of elite bananas (Musa acuminata, AAA) cultivars in four locations of Puerto Rico. The Journal of Agriculture of University of Puerto Rico 73(3):209-221.

Jaramillo, R. 1982. Las principales características morfológicas del fruto de banano, variedad Cavendish Gigante (Musa AAA) en Costa Rica. Unión de Países Exportadores de Banano. Panamá.

Johns, G. 1996. Effects of bunch trimming and double bunch covering on yield of bananas during winter in New South Wales. Australian Journal of Experimental Agriculture 36:229-235.

Lara, F. 1970. Problemas y procedimientos bananeros en la zona Atlántica de Costa Rica. Imprenta Trejos, San José, Costa Rica.

Meyer, J. 1975. Influence de l'ablation de mains sur le rendement en poids des régimes de bananes par catégories de conditionnent aux Antilles. Fruits 30(11):663-668.

Mora, M. 2000. Efecto de las prácticas de desmane y desdede sobre la producción y la calidad del fruto de banano (Musa AAA, cv. Gran Enano). Tesis Lic. Ing. Agr., Instituto Tecnológico de Costa Rica, San José, Costa Rica.

Robinson, JC., y V. Galán-Sauco. 2010. Bananas and plantains. Institute for Tropical and Subtropical Crops, South Africa. CAB International. $2^{\text {nd }}$ ed. Wallingford, UK.

Rodríguez, G., N. Muñoz, y J. Marquez. 2006. Poda de manos en el clon FHIA-21 (Musa AAAB) y su efecto sobre las dimensiones del fruto y aspectos de calidad. En: E. Soprano, F.A. Tcacenco, L.A. Litchemberg, y M.C. Silva, editores, XVII Reunâo International da Associaçâo nas Pesquisas sobre banano no Caribe e na América Tropical, ACORBAT, Santa Catarina, Brasil. p. 536-534.

SAS Institute. 2004. SAS/STAT®. User's guide: Statistics. Version 9.1. Cary, NC, USA.

Soto, M. 1992. Cosecha y empaque de la fruta. En: M. Soto, editor, Bananos: cultivo y comercialización. $2^{\text {nd }}$ ed. Litografía e Imprenta LIL S.A., San José, Costa Rica. p. 367-438.

Soto, M., E. Soto, P. Solís, y A. López. 1992. Siembra y operaciones de cultivo. En: M. Soto, editor, Bananos: 
cultivo y comercialización. $2^{\text {nd }}$ ed. Litografía e Imprenta LIL S.A., San José, Costa Rica. p. 211-365.

Vargas, A. 2001. Efecto de la intensidad de desmane sobre el peso del racimo de banano (Musa AAA, cvs. 'Gran Enano' y 'Valery') en el crecimiento y las dimensiones del fruto en dos épocas del año. CORBANA 27(54):13-34.

Vargas, A. 2002. Alta intensidad de desmane en banano (Musa AAA, cvs. Grande Naine y Williams), su efecto sobre el peso del racimo y las dimensiones de los frutos. CORBANA 28(55):27-42.

Vargas, A. 2003. Efecto de tres intensidades de desdede sobre el desarrollo del racimo de banano (Musa AAA, Cvs. Grande Naine y Valery). CORBANA 26(56): 27-38.

Vargas, A. 2006. Efecto de la retención de la última mano o de la remoción parcial o total de frutos de la primera mano sobre las características y el rendimiento de racimos de banano (Musa AAA). CORBANA 32(59):1-16.
Vargas, A. 2008. Efecto de la combinación de las prácticas de desmane y desgaje sobre el desarrollo del racimo de banano (Musa AAA, cv. Grande Naine). En: J. Sandoval, editor, Informe anual 2007. Corporación Bananera Nacional, San José, CR. 215 p.

Vargas, 2010. Efecto del desmane y de la modalidad de cosecha sobre las características y producción de racimos de plátano tipo Francés FHIA-21. Tropicultura 28(1):16-23.

Vargas,A., y F. Blanco. 2000.Consideraciones metodológicas para la evaluación del desmane en banano (Musa AAA, cv. 'Valery'). InfoMusa 9(2):19-21.

Vargas, A., y Blanco, F. 2004. Metodología para estimar la intensidad de desmane en racimos de banano (Musa AAA, cv. Valery). CORBANA 30(57):107-119.

Vargas, A., J. Sandoval, y F. Blanco. 1999. Efecto de la intensidad de desmane sobre las dimensiones y el fruto y peso del racimo de banano (Musa AAA cv. 'Valery'). CORBANA 24(51):85-92. 